\title{
Analysis of Torque Measurements on Films with Oblique Anistropy
}

\author{
Leon Abelmann, Vladimir Kamberský ${ }^{1}$, Cock Lodder, Theo J.A. Popma \\ MESA Research Insitute, University of Twente, P.O. Box 217, 7500 AE Enschede \\ ${ }^{1}$ On leave from Tsjech Institute of Physics, Prague
}

\begin{abstract}
A measurement method is discussed to determine the magnetic anisotropy energy in a sample without assuming an a priori model for the origins of the anisotropy. The measurement procedure involves torque measurements in five different planes. Since it is especially useful for films with an oblique anisotropy axis, the method is illustrated on an obliquely evaporated $\mathrm{Co}_{80} \mathrm{Ni}_{20}$ film.
\end{abstract}

\section{INTRODUCTION}

For certain magnetic recording applications metalevaporated (ME) tape can be used. In this type of media the film is deposited obliquely on a moving substrate. As a result the anisotropy axis is inclined with respect to the film normal. Analysing torque measurements on such films with an oblique anistropy axis is complex because measurements in several planes are necessary [1]. The common approach in analysis of the anistropy in these films is to assume a physical model based on a priori assumptions like the type of crystal structure and the direction of columns. Including second order crystal anistropy is not simple and the need for a more general model arises. Using spherical harmonics, it is possible to describe the anisotropy without $a$ priori assumptions on the origins of anisotropy. Taking torque measurements in five different planes, we can describe the magnetic anisotropy in second and fourth order for any arbitrary sample.

\section{THEORY}

The energy in the sample has to be described as function of the direction of the magnetization $\mathbf{M}$. This direction can be represented by the coordinates of the vector $m=M / M I$ or by the polar angles $(\theta, \varphi)$, see figure 1 . It is assumed that the sample has only anisotropy axes and no preferred directions:

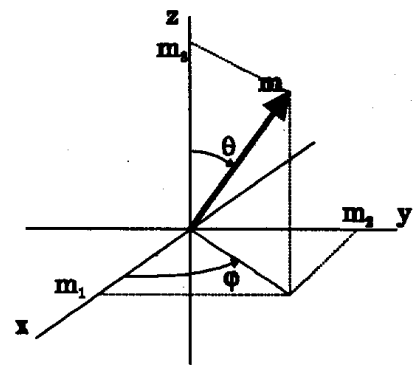

Fig. 1 Definition of coordinate system and representation of magnetization direction. The $x z$ plane corresponds with the film plane, the $x y$ plane with the vapour incidence plane

Manuscript received February 15, 1993. This reseanch is supported by the EC CAMST program.
$E(\mathbf{m})=E(-\mathbf{m})$. Then the general description of the anistropy energy in terms of coordinates of $m=\left[m_{1}, m_{2}, m_{3}\right]$

$$
\begin{aligned}
& \mathrm{E}(\mathbf{m})=\mathrm{a}_{0}+\sum_{\mathrm{i}=1}^{3} \sum_{\mathrm{i}=1}^{3} \mathrm{a}_{\mathrm{ij}}^{2} \mathrm{~m}_{\mathrm{j}} \mathrm{m}_{\mathrm{j}} \\
& +\sum_{\mathrm{i}=1}^{3} \sum_{\mathrm{j}=1}^{3} \sum_{\mathrm{k}=1}^{3} \sum_{\mathrm{l}=1}^{3} \mathrm{a}_{\mathrm{ijk} \mathbf{k}}^{2} \mathrm{~m}_{\mathrm{i}} \mathrm{m}_{\mathrm{j}} \mathrm{m}_{\mathbf{k}} \mathrm{m}_{1}+\ldots
\end{aligned}
$$

In this representation it takes 90 constants to describe the anisotropy energy in second and fourth order. Of course many of these constants are interdependent because of symmetry and $|\mathrm{m}|=1$. The analysis of torque curves using this representation is complicated because the basis functions $\left(m_{i} m_{j}, m_{i} m_{j} m_{k} m_{1}, \ldots\right)$ are not orthogonal.

These difficulties can be eliminated when one uses a description in terms of polar angles. In this representation the basis functions are the spherical harmonics $Y_{n, m}$, which are $3 \mathrm{D}$ equivalents of $\sin (n \theta)$ and $\cos (n \theta)$ used in onedimensional fourier analysis:

$$
\mathrm{E}(\theta, \varphi)=\sum_{\mathrm{n}=1}^{\infty} \sum_{\mathrm{m}=-2 \mathrm{n}}^{2 \mathrm{n}} \mathbf{k}_{2 \mathrm{n}, \mathrm{m}} \mathrm{Y}_{2 \mathrm{n}, \mathrm{m}}(\boldsymbol{\theta}, \varphi)
$$

Now only five constants are necessary to describe the anisotropy energy in second order, and nine additional constants for the fourth order.

For comparison with experimental data, we used the basis set of real spherical harmonics listed by Birrs [2]. $Y_{n, m l}$ and $Y_{n,-\operatorname{ml}}$ are proportional to $\cos (|\mathrm{m}| \varphi)$ and $\sin (|\mathrm{m}| \varphi)$ respectively. The coeficients in equations (2) and (4) and table II refer to this basis. For rotations of the coordinate system we transform this basis to a set of normalized complex spherical harmonics and used standard Wigner matrices [3].

In order to determine the anisotropy energy in the sample up to the fourth order, we have to determine fourteen anisotropy coeficients $k_{n, m}$. For this we need to take torque measurements in five different planes, as shown in table I.

TABLE I

Mrasurement Planes

\begin{tabular}{ccc}
\hline $\mathrm{Lx}_{\mathrm{a}}$ & $\theta\left[^{\circ}\right]$ & $\varphi\left[\left[^{\circ}\right]\right.$ \\
\hline $\mathrm{La}_{\mathrm{a}}$ & 90 & -90 to 90 \\
$\mathrm{Lb}$ & -90 to 90 & 0 \\
$\mathrm{Lc}$ & -90 to 90 & 90 \\
$\mathrm{Ld}$ & -90 to 90 & 45 \\
$\mathrm{Lc}$ & -90 to 90 & -45 \\
\hline
\end{tabular}

Each plane is defined by the path the applied field describes in the measurement coordinate system with angles as shown in figure 1.

We describe the torque measurements using sine and cosine terms $\left(P_{n}, Q_{n}\right)$ or amplitude and phase shift $\left(L_{n}, \alpha_{n}\right)$. 


$$
\begin{aligned}
& L_{x}(\gamma)= \\
& \sum_{n=1}^{2} P_{2 n, x} \cos (2 n \gamma)+Q_{2 n, x} \sin (2 n \gamma)= \\
& \sum_{n=1}^{2} L_{2 n, x} \sin \left[2 n\left(\gamma+\alpha_{2 n, x}\right)\right]
\end{aligned}
$$

With index $x$ refering to table $I$ and $\gamma$ the measurement angle (either $\theta$ or $\varphi$ ). These five measurements result in 20 fourier coeficients of which some are mutually dependent. The relation between the 20 fourier coefficients and the 14 anisotropy coeficients was solved analytically using Mathematica software.

\section{EXPERIMENT}

To illustrate this method we measured an obliquely evaporated $1.1 \mu \mathrm{m}$ thick $\mathrm{Co}_{80} \mathrm{Ni}_{20}$ film. The vapour incidence angle was $60^{\circ}$ from the substrate normal, the substrate $(5 \times 10 \mathrm{~mm})$ was glass and no oxygen was added during the process. For the measurements in the $L_{d}$ and $L_{e}$ plane (Table I) we used a holder cut under an angle of $45^{\circ}$. For practical reasons the coordinate system was chosen so that $\mathrm{xz}$ is the film plane and $\mathrm{xy}$ lies close to the incidence plane. In each measurent plane five field values between 1000 and $1600 \mathrm{kAm}^{-1}$ were taken. To correct for the finite field error the modulus $L_{n, x}$ (3) was plotted against $1 / \mathrm{H}^{2}$ or $1 / H$ for $n=2$ and $n=4$ respectively. Figure 2 gives an example. This gives a better estimation of the torque at infinite field than extrapolating $P_{n, x}$ and $Q_{n, x}$ coeficients

TABLE II

MEASURED ANISTROPY COEFICIENTS OF AN OBLIOUELY BVAPORATED Co80Ni20 FLM $\left[\mathrm{kJm}^{-3}\right]$

\begin{tabular}{ccc}
\hline $\mathrm{kn}, \mathrm{m}$ & $\mathrm{n}=2$ & $\mathrm{n}=4$ \\
\hline $\mathrm{m}=-4$ & & -0.10 \\
$\mathrm{~m}=-3$ & & -0.09 \\
$\mathrm{~m}=-2$ & 29.6 & -0.33 \\
$\mathrm{~m}=-1$ & 16.2 & -0.54 \\
$\mathrm{~m}=0$ & -71.1 & 6.00 \\
$\mathrm{~m}=1$ & -12.5 & -0.22 \\
$\mathrm{~m}=2$ & -62.0 & 0.27 \\
$\mathrm{~m}=3$ & & 0.02 \\
$\mathrm{~m}=4$ & & 0.08 \\
\hline
\end{tabular}

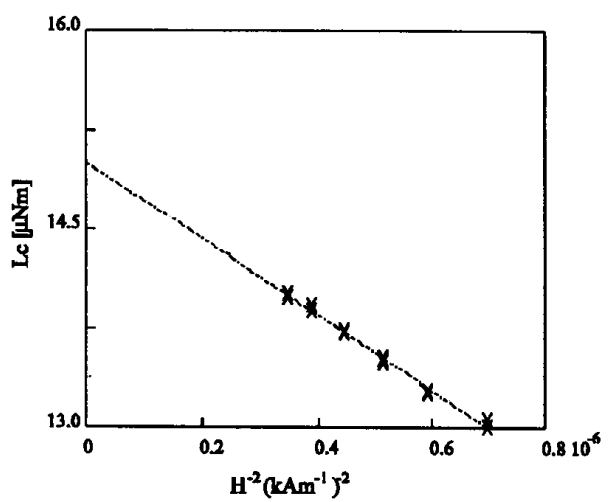

Fig. 2 Example of correction for finite field error (measurement $L c$ ) independently since the argument $\alpha_{n, x}$ is field-independent. Table II gives the resulting anisotropy coefficients.

Figure 3 shows the energy surface reconstructed from (2) for the sum of second and fourth order. The minimum of the energy surface was shifted to zero in order to obtain a reasonable image. This minimum is reached when the magnetisation lies close to the $x$ axis, which can be seen more clearly in the cross section in the xy plane (inset).

Figure 4 shows the energy surface of the fourth order

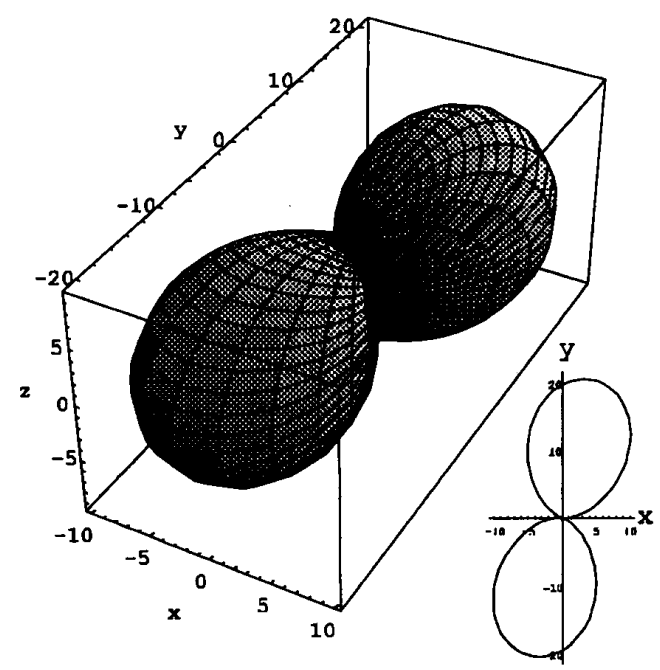

Fig. 3 Energy surface reconstructed from the torque measurements. The minimum energy is obtained when the magnetisation lies close to the $x$-axis, so this is a mainly in-plane sample. (Energy is shown in $\mu \mathrm{J}$ )

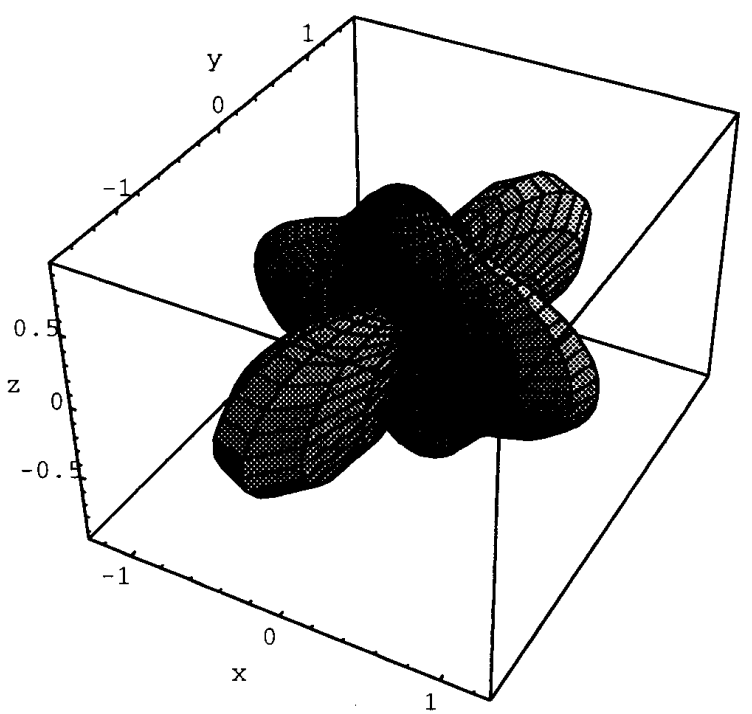

Fig. 4 Energy surface of fourth order components as calculated from torque measurement. This surface is correlated to the crystal structure in the sample. 
component only and therefore represents the preferred crystal orientation.

\section{ANALỴSIS}

In sufficiently high fields the only reason for the appearance of fourth order anisotropy coefficients $\left(k_{4, m}\right)$ is the presence of crystal anisotropy. Dipolar effects (like shape anisotropy e.g.) only contribute to the second order anisotropy coefficients $\left(k_{2, m}\right)$. Since different types of crystal structures and crystal orientations will cause different sets of fourth order coefficients it is in principle possible to obtain information on the crystal structure from the five torque measurements. The interpretation of the second and fourth order anisotropy coefficients will be treated separately.

Using $m=[\cos (\varphi) \sin (\theta), \sin (\varphi) \sin (\theta), \cos (\theta)]$ we can rewrite the second order part of (2) in a form analogous to (1), the $a_{i j}$ corresponding to the matrix elements:

$$
\begin{aligned}
& \mathrm{E}(\mathbf{m})=\dot{\mathbf{m}}^{\mathbf{T}} \mathbf{K}_{2} \mathbf{m} \\
& \mathbf{K}_{2}=\frac{3}{2}\left|\begin{array}{ccc}
2 \mathbf{k}_{2,2} & 2 \mathbf{k}_{2,-2} & \mathbf{k}_{2,1} \\
2 \mathbf{k}_{2,-2} & -2 \mathbf{k}_{2,2} & \mathbf{k}_{2,-1} \\
\mathbf{k}_{2,1} & \mathbf{k}_{2,-1} & \mathbf{k}_{2,0}
\end{array}\right|
\end{aligned}
$$

Diagonalisation of matrix $K_{2}$ yields the directions of the main second order anisotropy axes. Assume three second order anisotropy axis along the co-ordinate axis like in figure 5, then $K_{i}=-\left(K_{2}\right)_{i j}$. Energy is a relative quantity so that any arbitrary value $E_{0}$ can be added to it. Since $|m|=1$ this is equivalent to adding a $\mathrm{E}_{0}$ to the diagonal elements of $\mathbf{K}$. This allows us to choose one of the $K_{j}$ zero, e.g. $K_{2}$

Applying this method to the $\mathrm{Co}_{80} \mathrm{Ni}_{20}$ sample it is found that the incidence plane lies at an angle of $13.3^{\circ}$ from the $x$ axis (toward the $z$ axis). Along the $z$-axis lies a $K_{3}$ anisotropy axis with a value of $309 \mathrm{kJm}^{-3}$. In the incidence plane lies a $\mathrm{K}_{1}$ anisotropy axis with a value $419 \mathrm{kJm}^{-3}$ and inclined $12.6^{\circ}$ out of the film plane (from the $\mathrm{x}$-axis towards the $y$-axis).

The analysis of the fourth order part is far more complex since it is not obvious to find a diagonalisation procedure analogous to that used in the second order analysis. We can

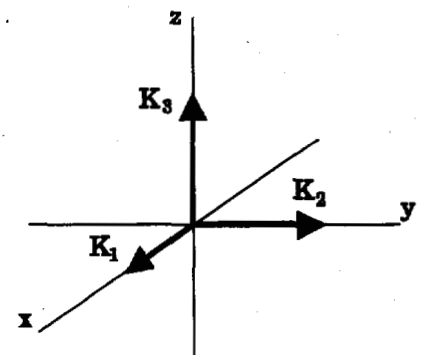

Fig. 5 After proper transformations the second order anisotropy can be described by three anisotropy axis along the coordinate system axis. One of the axis can be chosen zemo. however assume that the incidence plane found in that analysis is a mirror plane. If we now rotate the coordinate system so that the $z$ axis is perpendicular to the film plane and the $y$ axis lies within the incidence plane, we observe indeed that $k_{4,-m}$ nearly vanish. If we then rotate the coordinate system so that the $\mathrm{z}$-axis is perpendicular to the incidence plane we find that symmetry axis in the incidence plane is inclined $13^{\circ}$ from the film normal. We then identify our $\mathrm{z}$-axis with this symmetry axis and find a strong $\mathbf{k}_{\mathbf{4}, 0}$ value of $22 \mathrm{kJm}^{-3}$ next to a much smaller $\mathrm{k}_{4,2}$ and $\mathbf{k}_{4,4}$.

The strong $\mathbf{k}_{\mathbf{4}, 0}$ value can be identified with a hexagonal crystal structure with the main orientation of the c-axis inclined $13^{\circ}$ from the film normal (whereas the second order anisotropy axis was inclined $12.6^{\circ}$ out of the film plane). The value of the second order crystal anistropy constant $\mathrm{K}_{2}$ is $11 / 3 \mathrm{k}_{4,0}=113 \mathrm{kJm}^{-3}$ (neglecting $\mathrm{K}_{3}$ ). The other anisotropy coefficients could be caused by uncertainties in the extrapolation method used to determine the torque fourier coefficients or by the presence of more c-axis orientations. It is unlikely that the $\mathrm{k}_{4,4}$ value is caused by a cubic crystal structure since in that case it would have its $<100>$ axis along the $\mathrm{z}$-axis.

\section{CONCLUSIONS}

By taking torque measurements in five different planes the magnetic anisotropy energy in an arbitrary sample can be determined up to the fourth order without assuming an $a$ priori model for the origin of the anisotropy. An obliquely evaporated $\mathrm{Co}_{80} \mathrm{Ni}_{20}$ was used to illustrate the method.

The second order part of the energy can be easily analysed as a system of two mutually perpendicular second order anisotropy axes. In the $\mathrm{Co}_{80} \mathrm{Ni}_{20}$ we found one axis in-plane and perpendicular to the incidence plane and one in the incidence plane slightly inclined out of the film plane. The fourth order part is more difficult to analyse because of the lack of a diagonalisation procedure. In the $\mathrm{Co}_{80} \mathrm{Ni}_{20}$ film mainly the influence of a hexagonal texture with inclined caxis was found.

\section{REFERENCES}

[1] S. Swaving; G.J. Gerritsma, J.C. Lodder, Th.J.A Popma, "3D analysis of magnetic anisotropies in $\mathrm{OI}$ and CVI films," J. Magn. Magn. Mat., vol 67 pp. 155-64, 1987.

[2] Robert R. Birrs, Symmetry and Magnetism, Amsterdam: Nonth-Holland, 1964.

[3] D.A. Varshalovich, A.N. Moskalev, V.K. Khersonskii, Kvantovaya teoria uglovogo momenta, Leningrad: Nauka, 1975 (in Russian) 